\title{
EXISTENCE AND ULAM-HYERS STABILITY OF THE IMPLICIT FRACTIONAL BOUNDARY VALUE PROBLEM WITH $\psi$-CAPUTO FRACTIONAL DERIVATIVE
}

\author{
Hanan A. Wahash ${ }^{1}$, Mohammed S. Abdo ${ }^{1,2}$, Satish K. Panchal ${ }^{3}$ \\ ${ }^{1}$ Research Scholar at Department of Mathematics, Dr. Babasaheb Ambedkar Marathwada University \\ Aurangabad 431004 (M.S.), India \\ ${ }^{2}$ Department of Mathematics, Hodeidah University \\ Al-Hodeidah, Yemen \\ ${ }^{3}$ Department of Mathematics, Dr. Babasaheb Ambedkar Marathwada University \\ Aurangabad 431004 (M.S.), India \\ hawahash86@gmail.com,msabdo1977@gmail.com,drpanchalsk@gmail.com
}

Received: 9 September 2019; Accepted: 30 January 2020

\begin{abstract}
In this paper, we investigate the existence, uniqueness and Ulam-Hyers stability of solutions for nonlinear implicit fractional differential equations with boundary conditions involving a $\psi$-Caputo fractional derivative. The obtained results for the proposed problem are proved under a new approach and minimal assumptions on the function $f$. The analysis is based upon the reduction of the problem considered to the equivalent integral equation, while some fixed point theorems of Banach and Schauder and generalized Gronwall inequality are employed to obtain our results for the problem at hand. Finally, the investigation is illustrated by providing a suitable example.
\end{abstract}

MSC 2010: 34A08, 26A33, 34A12, 47H10

Keywords: fractional differential equations, $\psi$-fractional integral and derivative, existence and Ulam-Hyers stability, fixed point theorem

\section{Introduction}

Fractional calculus studies the differentiation and integration to fractional order. It is considered as a generalization of classical calculus. The fractional differential equations (FDEs) have become an emerging area of recent research in science, engineering and mathematics [1-4]. So, in the literature, there are several studies covering comparable topics to distinct operators such as [1,5-11] and the references cited therein. The stability of functional equations was originally raised by Ulam [12] and next by Hyers [13]. Thereafter, this kind of stability is called the Ulam-Hyers stability. For some recent results of stability analysis by different types of operators, we refer the reader to a series of papers [14-17], and the references are given therein. Recently, Almeida in [18], presented a new type of fractional 
differentiation operator called the $\psi$-Caputo fractional operator and extended the work of the Caputo [2,4].

This article is motivated by the importance of implicit classical differential equations $f\left(t, u(t), u^{\prime}(t), \ldots, u^{(n-1)}(t)\right)=0$, the implicit fractional differential equation (IFDE) involving a Caputo fractional derivative of the form

$$
{ }^{c} \mathscr{D}_{a^{+}}^{\alpha} u(t)=f\left(t, u(t),{ }^{c} \mathscr{D}_{a^{+}}^{\alpha} u(t)\right), t>a,
$$

and fractional differential equation (FDE) involving the $\psi$-Caputo fractional derivative of the form

$$
{ }^{c} \mathscr{D}_{a^{+}}^{\alpha ; \psi} u(t)=f(t, u(t)), t \in[a, b],
$$

under various (initial/boundary/nonlocal) conditions. The problem (1) has been discussed by many researchers, see [9,15,19-22], by the use of different fixed point techniques. Very recently, the problem (2) has been studied by Almeida et al., in [23,24], Vivek et al. [25], and Abdo et al. [26] under different conditions and techniques. For instance, in [24], the authors investigated the existence and uniqueness of a solution of the initial value problem (IVP) for a nonlinear FDE involving the $\psi-$ Caputo fractional derivative

$$
\begin{gathered}
{ }^{c} \mathscr{D}_{a^{+}}^{\alpha ; \psi} u(t)=f(t, u(t)), \quad n-1<\alpha<n, \\
u(a)=u_{a}, u_{\psi}^{[k]}(a)=u_{a}^{k}, k=1, \ldots n-1,
\end{gathered}
$$

where ${ }^{c} \mathscr{D}_{a^{+}}^{\alpha ; \psi}$ is the $\psi$-Caputo fractional derivative, $u_{a}, u_{a}^{k} \in \mathbb{R}, t \in[a, b], u \in C^{n-1}[a, b]$ and $f:[a, b] \times \mathbb{R} \longrightarrow \mathbb{R}$ is a continuous function.

Motivated by the above works, we prove the existence, uniqueness, and Ulam-Hyers stability of the nonlinear implicit fractional differntial equation with boundary conditions and $\psi$-Caputo fractional derivatives of the form

$$
\begin{gathered}
{ }^{c} \mathscr{D}_{a^{+}}^{\alpha ; \psi} u(t)=f\left(t, u(t),{ }^{c} \mathscr{D}_{a^{+}}^{\alpha ; \psi} u(t)\right), t \in[a, b], \\
u_{\psi}^{[k]}(a)=u_{a}^{k}, k=0,1, \ldots, n-2 ; u_{\psi}^{[n-1]}(b)=u_{b},
\end{gathered}
$$

where ${ }^{c} \mathscr{D}_{a^{+}}^{\alpha ; \psi}$ is the $\psi$-Caputo fractional derivative of order $n-1<\alpha \leq n$ ( $n=$ $=[\alpha]+1), f:[a, b] \times \mathbb{R} \times \mathbb{R} \longrightarrow \mathbb{R}$ is given a continuous function, $u_{a}^{k}, u_{b}$ are fixed reals $(k=0,1, \ldots, n-2)$ and $u \in C^{n-1}[a, b]$ such that ${ }^{c} \mathscr{D}_{a^{+}}^{\alpha ; \psi} u$ exists and is continuous in $[a, b]$. Also, we denote $u_{a}^{0}=u_{a}$. 


\section{Preliminaries}

In this fragment, let us recall some basic definitions, lemmas, and results related to the Caputo fractional derivative with respect to another function $([2,18,24])$ which are used throughout this paper.

Definition 1 ( $\psi$-Riemann-Liouville fractional operators [24]). Let $\alpha>0, h$ an integrable function defined on finite or infinite interval $[a, b]$ and $\psi \in C^{1}[a, b]$ an increasing function such that $\psi^{\prime}(t) \neq 0$, for all $t \in[a, b]$. Then the left sided fractional integrals and fractional derivatives of order $\alpha$ of a function $h$ with respect to another function $\psi$ are defined as

$$
\mathscr{I}_{a^{+}}^{\alpha ; \psi} h(t)=\frac{1}{\Gamma(\alpha)} \int_{a}^{t} \psi^{\prime}(s)[\psi(t)-\psi(s)]^{\alpha-1} h(s) d s, t>a,
$$

and

$$
\mathscr{D}_{a^{+}}^{\alpha ; \psi} h(t)=\left[\frac{1}{\psi^{\prime}(t)} \frac{d}{d t}\right]^{n} \mathscr{I}_{a^{+}}^{n-\alpha, \psi} h(t), t>a,
$$

respectively, where $\Gamma(\cdot)$ is a gamma funcion and $n=[\alpha]+1,[\alpha]$ denotes the integer part of the real number $\alpha$.

Definition 2 ( $\psi$-Caputo fractional derivative [24]). Let $\alpha>0$, and $h, \psi \in C^{n-1}[a, b]$ two functions such that $\psi$ is an increasing function and $\psi^{\prime}(t) \neq 0$, for all $t \in[a, b]$. Then the left sided $\psi$-Caputo fractional derivative of function $h$ of order $\alpha$ is determined as

$$
{ }^{c} \mathscr{D}_{a^{+}}^{\alpha ; \psi} h(t)=\mathscr{D}_{a^{+}}^{\alpha ; \psi}\left[h(t)-\sum_{k=0}^{n-1} \frac{h_{\psi}^{[k]}(a)}{k !}[\psi(t)-\psi(a)]^{k}\right] .
$$

where $h_{\psi}^{[k]}(t)=\left[\frac{1}{\psi^{\prime}(t)} \frac{d}{d t}\right]^{k} h(t)$ and $n=[\alpha]+1$ for $\alpha \notin \mathbb{N}, n=\alpha$ for $\alpha \in \mathbb{N}$.

Lemma 1 [24] Let $n-1<\alpha<n(\alpha \notin \mathbb{N})$, and $h \in C^{n}[a, b]$. Then we have

${ }^{c} \mathscr{D}_{a^{+}}^{\alpha ; \psi} h(t)=\mathscr{I}_{a^{+}}^{n-\alpha, \psi}\left[\frac{1}{\psi^{\prime}(t)} \frac{d}{d t}\right]^{n} h(t)=\frac{1}{\Gamma(n-\alpha)} \int_{a}^{t} \psi^{\prime}(s)[\psi(t)-\psi(s)]^{n-\alpha-1} h_{\psi}^{[n]}(s) d s$.

In particular, if $\alpha=n \in \mathbb{N}$, one has ${ }^{c} \mathscr{D}_{a^{+}}^{\alpha ; \psi} h(t)=h_{\psi}^{[n]}(t)$.

Lemma $2[18,24]$ Let $\alpha>0$. The following holds:

1. If $h \in C[a, b]$, then ${ }^{c} \mathscr{D}_{a^{+}}^{\alpha ; \psi} \mathscr{I}_{a^{+}}^{\alpha ; \psi} h(t)=h(t)$.

2. $h \in C^{n-1}[a, b]$, then $\mathscr{I}_{a^{+}}^{\alpha ; \psi}{ }^{c} \mathscr{D}_{a^{+}}^{\alpha ; \psi} h(t)=h(t)-\sum_{k=0}^{n-1} \frac{h_{\psi}^{[k]}(a)}{k !}[\psi(t)-\psi(a)]^{k}$. 
Lemma $3[2,18]$ Let $\alpha>0$ and $h:[a, b] \rightarrow \mathbb{R}$. Then we have

$$
\begin{aligned}
& \text { 1. } \mathscr{I}_{a^{+}}^{\alpha ; \psi}[\psi(t)-\psi(a)]^{\beta-1}=\frac{\Gamma(\beta)}{\Gamma(\alpha+\beta)}[\psi(t)-\psi(a)]^{\alpha+\beta-1}, \beta>0 . \\
& \text { 2. }{ }^{c} \mathscr{D}_{a^{+}}^{\alpha ; \psi}[\psi(t)-\psi(a)]^{\beta-1}=\frac{\Gamma(\beta)}{\Gamma(\beta-\alpha)}[\psi(t)-\psi(a)]^{\beta-\alpha-1}, \beta>n \in \mathbb{N} . \\
& \text { 3. }{ }^{c} \mathscr{D}_{a^{+}}^{\alpha ; \psi}[\psi(t)-\psi(a)]^{k}=0, \forall k \in\{0,1, \ldots, n-1\}, n \in \mathbb{N} . \\
& \text { 4. } \mathscr{I}_{a^{+}}^{\alpha ; \psi} \mathscr{I}_{a^{+}}^{\beta, \psi} h(t)=\mathscr{I}_{a^{+}}^{\alpha+\beta ; \psi} h(t), \beta>0 . \\
& \text { 5. }{ }^{c} \mathscr{D}_{a^{+}}^{\alpha ; \psi} C=0, \text { for any constant } C .
\end{aligned}
$$

\section{Main results}

Before stating and proving the main results, we given the following Lemma.

Lemma 4 [26] Let $n-1<\alpha<n$ and $g$ an integrable function. Then a function $u \in C^{n-1}[a, b]$ is a solution of the fractional boundary value problem

$$
\begin{gathered}
{ }^{c} \mathscr{D}_{a^{+}}^{\alpha ; \psi} u(t)=g(t), \quad t \in[a, b], \\
u_{\psi}^{[k]}(a)=u_{a}^{k}, k=0,1, \ldots, n-2 ; u_{\psi}^{[n-1]}(b)=u_{b},
\end{gathered}
$$

if and only if $u(t)$ satisfies the following fractional integral equation

$$
\begin{aligned}
u(t)= & \frac{1}{\Gamma(\alpha)} \int_{a}^{t} \psi^{\prime}(s)[\psi(t)-\psi(s)]^{\alpha-1} g(s) d s+\left[\frac{u_{b}}{(n-1) !}\right. \\
& \left.+\frac{g(a)[\psi(b)-\psi(a)]^{\alpha-n+1}}{(n-2) ! \Gamma(\alpha-n+2)}\right][\psi(t)-\psi(a)]^{n-1}-\frac{[\psi(t)-\psi(a)]^{n-1}}{(n-1) ! \Gamma(\alpha-n+1)} \\
& \times \int_{a}^{b} \psi^{\prime}(s)[\psi(b)-\psi(s)]^{\alpha-n} g(s) d s+\sum_{k=0}^{n-2} \frac{u_{a}^{k}}{k !}[\psi(t)-\psi(a)]^{k}
\end{aligned}
$$

\subsection{Uniqueness result via Banach's fixed point theorem}

Theorem 1 Assume that $f:[a, b] \times \mathbb{R} \times \mathbb{R} \rightarrow \mathbb{R}$ is continuous and there exists a positive constant $0<\eta \neq 1$ such that

$$
\left|f(t, x, y)-f\left(t, x^{*}, y^{*}\right)\right| \leq \eta\left[\left|x-x^{*}\right|+\left|y-y^{*}\right|\right],
$$


for each $t \in[a, b]$, and $x, y, x^{*}, y^{*} \in \mathbb{R}$. If

$$
\mathbb{W}:=\left(\frac{1}{\Gamma(\alpha+1)}+\frac{[\psi(b)-\psi(a)]+n-1}{(n-1) ! \Gamma(\alpha-n+2)}\right) \frac{\eta}{1-\eta}[\psi(b)-\psi(a)]^{\alpha}<1 .
$$

Then the implicit fractional BVP (5)-(6) has a unique solution on $[a, b]$.

ProOF Let

$$
\begin{gathered}
{ }^{c} \mathscr{D}_{a^{+}}^{\alpha ; \psi} u(t)=F_{u}(t), t \in[a, b], \\
u_{\psi}^{[k]}(a)=u_{a}^{k}, k=0,1, \ldots, n-2, u_{\psi}^{[n-1]}(b)=u_{b},
\end{gathered}
$$

where $F_{u}(t):=f\left(t, u(t), F_{u}(t)\right)$. Set

$$
\Omega:=\left\{u \in C^{n-1}[a, b]:{ }^{c} \mathscr{D}_{a^{+}}^{\alpha ; \psi} u \in C[a, b] ; t \in[a, b]\right\} .
$$

In order to transform the problem (12)-(13) into a fixed point problem, we introduce an operator $\mathscr{T}: \Omega \rightarrow \Omega$ by Lemma 4 as follows

$$
\begin{aligned}
(\mathscr{T} u)(t)= & \frac{1}{\Gamma(\alpha)} \int_{a}^{t} \psi^{\prime}(s)\left[\psi(t)-\psi(s){ }^{\alpha-1} F_{u}(s) d s+\left[\frac{u_{b}}{(n-1) !}\right.\right. \\
& \left.+\frac{F_{u}(a)[\psi(b)-\psi(a)]^{\alpha-n+1}}{(n-2) ! \Gamma(\alpha-n+2)}\right][\psi(t)-\psi(a)]^{n-1}-\frac{[\psi(t)-\psi(a)]^{n-1}}{(n-1) ! \Gamma(\alpha-n+1)} \\
& \times \int_{a}^{b} \psi^{\prime}(s)[\psi(b)-\psi(s)]^{\alpha-n} F_{u}(s) d s+\sum_{k=0}^{n-2} \frac{u_{a}^{k}}{k !}[\psi(t)-\psi(a)]^{k}
\end{aligned}
$$

We first show that $\mathscr{T}$ is well defined, i.e. $\mathscr{T}(\Omega) \subseteq \Omega$. To this end, we suppose $u \in C^{n-1}[a, b]$. It is obvious that $\mathscr{T} u \in C^{n-1}[a, b]$. Also, by (15) and Lemma 3, we get ${ }^{c} \mathscr{D}_{a^{+}}^{\alpha ; \psi}(\mathscr{T} u)(t)={ }^{c} \mathscr{D}_{a^{+}}^{\alpha ; \psi} \mathscr{I}_{a^{+}}^{\alpha ; \psi} F_{u}(t)$. Since the function $F_{u}(\cdot)$ is continuous on $[a, b]$, the Lemma 4 shows that

$$
{ }^{c} \mathscr{D}_{a^{+}}^{\alpha ; \psi}(\mathscr{T} u)(t)=F_{u}(t) .
$$

Therefore, ${ }^{c} \mathscr{D}_{a^{+}}^{\alpha: \psi}(\mathscr{T} u) \in C[a, b]$ i.e. $\mathscr{T} u \in \Omega$. Next, we shall apply the Banach fixed point theorem to verify that $\mathscr{T}$ defined by (15) has a fixed point. We just need to show that $\mathscr{T}$ is a contraction map in $\Omega$. Indeed, for $u_{1}, u_{2} \in \Omega$ and for each $t \in[a, b]$, we obtain

$$
\begin{aligned}
& \left|\mathscr{T} u_{1}(t)-\mathscr{T} u_{2}(t)\right| \\
\leq & \mathscr{I}_{a^{+}}^{\alpha ; \psi}\left|F_{u_{1}}(t)-F_{u_{2}}(t)\right|+\frac{[\psi(b)-\psi(a)]^{\alpha-n+1}[\psi(t)-\psi(a)]^{n-1}}{(n-2) ! \Gamma(\alpha-n+2)} \\
& \times\left|F_{u_{1}}(a)-F_{u_{2}}(a)\right|+\frac{[\psi(t)-\psi(a)]^{n-1}}{(n-1) !} \mathscr{I}_{a^{+}}^{\alpha-n+1 ; \psi}\left|F_{u_{1}}(b)-F_{u_{2}}(b)\right| .
\end{aligned}
$$


From our assumption, we obtain

$$
\left|F_{u_{1}}(t)-F_{u_{2}}(t)\right| \leq \frac{\eta}{1-\eta}\left\|u_{1}-u_{2}\right\| .
$$

By invoking the relation (18) into (17), we get

$$
\begin{aligned}
& \left\|\mathscr{T} u_{1}-\mathscr{T} u_{2}\right\| \\
\leq & \left(\frac{1}{\Gamma(\alpha+1)}+\frac{[\psi(b)-\psi(a)]+n-1}{(n-1) ! \Gamma(\alpha-n+2)}\right) \frac{\eta}{1-\eta}[\psi(b)-\psi(a)]^{\alpha}\left\|u_{1}-u_{2}\right\| \\
= & \mathbb{W}\left\|u_{1}-u_{2}\right\| .
\end{aligned}
$$

As $\mathbb{W}<1, \mathscr{T}$ is a contraction mapping. In view of the Banach fixed point theorem, $u$ is the unique solution to the problem (5)-(6) on $[a, b]$. The proof is completed.

\subsection{Existence result via Schauder's fixed point theorem}

Theorem 2 Assume that $f:[a, b] \times \mathbb{R} \times \mathbb{R} \rightarrow \mathbb{R}$ is continuous and there exist two positive constants $k_{0}$ and $k_{1}$ with $0<k_{1}<1$ such that

$$
|f(t, x, y)| \leq k_{0}|x|+k_{1}|y|, \forall(t, x, y) \in[a, b] \times \mathbb{R} \times \mathbb{R} .
$$

If

$$
\frac{k_{0}}{1-k_{1}}[\psi(b)-\psi(a)]^{\alpha}\left[\frac{1}{\Gamma(\alpha+1)}+\frac{n}{(n-1) ! \Gamma(\alpha-n+2)}\right]<\frac{1}{2} .
$$

Then the implicit fractional BVP (5)-(6) has at least one solution on $[a, b]$.

Proof Consider the set $\Omega$ and the operator $\mathscr{T}: \Omega \rightarrow \Omega$ defined by (14) and (15), respectively. The hypothesis of Schauder's fixed point theorem will be verified in several steps.

Step 1. The operator $\mathscr{T}$ is continuous.

Let $\left\{u_{n}\right\}_{n \in \mathbb{N}}$ be a sequence such that $u_{n} \rightarrow u$ in $\Omega$, as $n \rightarrow \infty$. Then for every $t \in[a, b]$, we have

$$
\begin{aligned}
& \left|\mathscr{T} u_{n}(t)-\mathscr{T} u(t)\right| \\
\leq & \mathscr{I}_{a^{+}}^{\alpha ; \psi}\left|F_{u_{n}}(t)-F_{u}(t)\right|+\frac{[\psi(b)-\psi(a)]^{\alpha-n+1}[\psi(t)-\psi(a)]^{n-1}}{(n-2) ! \Gamma(\alpha-n+2)} \\
& \times\left|F_{u_{n}}(a)-F_{u}(a)\right|+\frac{[\psi(t)-\psi(a)]^{n-1}}{(n-1) !} \mathscr{I}_{a^{+}}^{\alpha-n+1 ; \psi}\left|F_{u_{n}}(b)-F_{u}(b)\right|,
\end{aligned}
$$

which implies

$$
\left\|\mathscr{T} u_{n}-\mathscr{T} u\right\| \leq\left\|F_{u_{n}}(\cdot)-F_{u}(\cdot)\right\|\left[\frac{[\psi(b)-\psi(a)]^{\alpha}}{\Gamma(\alpha+1)}+\frac{n[\psi(b)-\psi(a)]^{\alpha}}{(n-1) ! \Gamma(\alpha-n+2)}\right] .
$$


Since $F_{u}(\cdot)$ is a continuous and $u_{n} \rightarrow u$, it follows that $\left\|\mathscr{T} u_{n}-\mathscr{T} u\right\| \rightarrow 0$ as $n \rightarrow \infty$, which implies that $\mathscr{T}$ is continuous.

Step 2. The operator $\mathscr{T}$ maps bounded sets into uniformly bounded sets in $\Omega$.

In fact, it is enough to show that for any $r>0$, there exists some $r^{\prime}>0$ such that for each $u \in \mathbb{B}_{r}:=\{u \in \Omega:\|u\| \leq r\}$, we have $\|\mathscr{T} u\| \leq r^{\prime}$.

Let $u \in \mathbb{B}_{r}$ and for each $t \in[a, b]$, we have

$$
\begin{aligned}
|(\mathscr{T} u)(t)| & \leq \frac{1}{\Gamma(\alpha)} \int_{a}^{t} \psi^{\prime}(s)[\psi(t)-\psi(s)]^{\alpha-1}\left|F_{u}(s)\right| d s+\left[\frac{\left|u_{b}\right|}{(n-1) !}\right. \\
& \left.\left.+\frac{\left|F_{u}(a)\right|[\psi(b)-\psi(a)]^{\alpha-n+1}}{(n-2) ! \Gamma(\alpha-n+2)}[\psi] b\right)-\psi(a)\right]^{n-1}+\frac{[\psi(b)-\psi(a)]^{n-1}}{(n-1) ! \Gamma(\alpha-n+1)} \\
& \times \int_{a}^{b} \psi^{\prime}(s)[\psi(b)-\psi(s)]^{\alpha-n}\left|F_{u}(s)\right| d s+\sum_{k=0}^{n-2} \frac{\left|u_{a}^{k}\right|}{k !}[\psi(t)-\psi(a)]^{k} .
\end{aligned}
$$

With the aid of our assumption, and definition of $F_{u}$, it is easy to get

$$
\left|F_{u}(t)\right| \leq \frac{k_{0}}{1-k_{1}}\|u\| \leq \frac{k_{0} r}{1-k_{1}} .
$$

Consequently,

$$
\begin{aligned}
\|\mathscr{T} u\| \leq & \frac{k_{0} r}{1-k_{1}}[\psi(b)-\psi(a)]^{\alpha}\left[\frac{1}{\Gamma(\alpha+1)}+\frac{n}{(n-1) ! \Gamma(\alpha-n+2)}\right] \\
& +\frac{\left|u_{b}\right|[\psi(b)-\psi(a)]^{n-1}}{(n-1) !}+\sum_{k=0}^{n-2} \frac{\left|u_{a}^{k}\right|}{k !}[\psi(b)-\psi(a)]^{k}:=r^{\prime} .
\end{aligned}
$$

So $\{\mathscr{T} u\}$ is a uniformly bounded set.

Step 3. The operator $\mathscr{T}$ maps bounded sets into equicontinuous sets of $\Omega$.

Let $\mathbb{B}_{r}$ be a bounded set of $\Omega$ defined as in step $2, t_{1}, t_{2} \in[a, b]$, with $t_{1}<t_{2}$, and let $u \in \mathbb{B}_{r}$. Then

$$
\begin{aligned}
& \left|(\mathscr{T} u)\left(t_{2}\right)-(\mathscr{T} u)\left(t_{1}\right)\right| \\
\leq & \frac{k_{0} r}{1-k_{1}} \frac{1}{\Gamma(\alpha)} \int_{a}^{t_{1}} \psi^{\prime}(s)\left[\left[\psi\left(t_{2}\right)-\psi(s)\right]^{\alpha-1}-\left[\psi\left(t_{1}\right)-\psi(s)\right]^{\alpha-1}\right] d s \\
& +\frac{k_{0} r}{1-k_{1}} \frac{1}{\Gamma(\alpha)} \int_{t_{1}}^{t_{2}} \psi^{\prime}(s)\left[\psi\left(t_{2}\right)-\psi(s)\right]^{\alpha-1} d s+\left[\frac{\left|u_{b}\right|}{(n-1) !}\right. \\
& \left.+\frac{k_{0} r[\psi(b)-\psi(a)]^{\alpha-n+1}}{\left(1-k_{1}\right)(n-2) ! \Gamma(\alpha-n+2)}\right]\left[\left[\psi\left(t_{2}\right)-\psi(a)\right]^{n-1}-\left[\psi\left(t_{1}\right)-\psi(a)\right]^{n-1}\right]
\end{aligned}
$$




$$
\begin{aligned}
& +\frac{k_{0} r\left[\left[\psi\left(t_{2}\right)-\psi(a)\right]^{n-1}-\left[\psi\left(t_{1}\right)-\psi(a)\right]^{n-1}\right]}{\left(1-k_{1}\right)(n-1) ! \Gamma(\alpha-n+1)} \int_{a}^{b} \psi^{\prime}(s)[\psi(b)-\psi(s)]^{\alpha-n} d s \\
& +\sum_{k=0}^{n-2} \frac{\left|u_{a}^{k}\right|}{k !}\left[\left[\psi\left(t_{2}\right)-\psi(a)\right]^{k}-\left[\psi\left(t_{1}\right)-\psi(a)\right]^{k}\right] \\
& \leq \frac{k_{0} r}{\left(1-k_{1}\right) \Gamma(\alpha+1)}\left[\left[\psi\left(t_{2}\right)-\psi(a)\right]^{\alpha}-\left[\psi\left(t_{2}\right)-\psi\left(t_{1}\right)\right]^{\alpha}-\left[\psi\left(t_{1}\right)-\psi(a)\right]^{\alpha}\right] \\
& +\frac{k_{0} r}{\left(1-k_{1}\right) \Gamma(\alpha+1)}\left[\psi\left(t_{2}\right)-\psi\left(t_{1}\right)\right]^{\alpha}+\sum_{k=0}^{n-2} \frac{\left|u_{a}^{k}\right|}{k !}\left[\left[\psi\left(t_{2}\right)-\psi(a)\right]^{k}-\left[\psi\left(t_{1}\right)-\psi(a)\right]^{k}\right] \\
& +\left[\frac{\left|u_{b}\right|}{(n-1) !}+\frac{k_{0} r n[\psi(b)-\psi(a)]^{\alpha-n+1}}{\left(1-k_{1}\right)(n-1) ! \Gamma(\alpha-n+2)}\right]\left[\left[\psi\left(t_{2}\right)-\psi(a)\right]^{n-1}-\left[\psi\left(t_{1}\right)-\psi(a)\right]^{n-1}\right]
\end{aligned}
$$

which tends to zero as $t_{1} \rightarrow t_{2}$, independent of $u$. Therefore $\mathscr{T}\left(\mathbb{B}_{r}\right)$ is equicontinuous in $\Omega$. So $\mathscr{T}$ is relatively compact on $\mathbb{B}_{r}$. By the Arzela-Ascoli theorems, $\mathscr{T}\left(\mathbb{B}_{r}\right)$ contained in a compact set, hence $\mathscr{T}: \Omega \rightarrow \Omega$ is continuous and completely continuous. To apply Schauder's fixed point theorem, we need to verify that there exists a closed convex bounded subset $\mathbb{B}_{\varepsilon}$ in $\Omega$ such that $\mathscr{T} \mathbb{B}_{\varepsilon} \subseteq \mathbb{B}_{\varepsilon}$. To this end, there exists a constant $\varepsilon>0$ such that

$$
\varepsilon \geq 2\left(\frac{\left|u_{b}\right|[\psi(b)-\psi(a)]^{n-1}}{(n-1) !}+\sum_{k=0}^{n-2} \frac{\left|u_{a}^{k}\right|}{k !}[\psi(b)-\psi(a)]^{k}\right) .
$$

Define $\mathbb{B}_{\varepsilon}=\{u \in \Omega:\|u\| \leq \varepsilon\} \subseteq \Omega$. It is clear that $\mathbb{B}_{\varepsilon}$ is closed, convex and bounded subsets of $\Omega$. By (21), (22), and inequalities (20), (23), then for every $u \in \mathbb{B}_{\varepsilon}$ and $t \in[a, b]$, we have

$$
\begin{aligned}
|\mathscr{T} u(t)| \leq & \frac{k_{0} \varepsilon}{1-k_{1}}[\psi(b)-\psi(a)]^{\alpha}\left[\frac{1}{\Gamma(\alpha+1)}+\frac{n}{(n-1) ! \Gamma(\alpha-n+2)}\right] \\
& +\frac{\left|u_{b}\right|[\psi(b)-\psi(a)]^{n-1}}{(n-1) !}+\sum_{k=0}^{n-2} \frac{\left|u_{a}^{k}\right|}{k !}[\psi(b)-\psi(a)]^{k} \leq \frac{\varepsilon}{2}+\frac{\varepsilon}{2}=\varepsilon .
\end{aligned}
$$

It follows that $\|\mathscr{T} u\| \leq \varepsilon$ for all $u \in \mathbb{B}_{\varepsilon}$, and hence $\mathscr{T} \mathbb{B}_{\varepsilon} \subseteq \mathbb{B}_{\varepsilon}$.

An application of Schauder's fixed point theorem shows that there exists at least a fixed point $u$ of $\mathscr{T}$ in $\Omega$. This fixed point $u$ is the solution to (5)-(6) on $[a, b]$, and the proof is completed.

\subsection{Ulam-Hyers stability}

This part is devoted to proving the Ulam-Hyers and generalized Ulam-Hyers stability of solution to the problem (5)-(6). 
Definition 3 The equation (5) is Ulam-Hyers stable if there exists a real number $\lambda_{f}>0$ with the following property: For every $\varepsilon>0, \widetilde{u} \in C^{n-1}[a, b]$, if

$$
\left|{ }^{c} \mathscr{D}_{a^{+}}^{\alpha, \psi} \widetilde{u}(t)-f\left(t, \widetilde{u}(t), F_{\widetilde{u}}(t)\right)\right| \leq \varepsilon,
$$

then there exists $u \in C^{n-1}[a, b]$ satisfying

$$
\begin{gathered}
\mathscr{D}_{a^{+}}^{\alpha, \psi} u(t)=f\left(t, u(t), F_{u}(t)\right), \quad t \in[a, b], \\
u_{\psi}^{[k]}(a)=\widetilde{u}_{\psi}^{[k]}(a), k=0,1, \ldots, n-2 ; u_{\psi}^{[n-1]}(b)=\widetilde{u}_{\psi}^{[n-1]}(b),
\end{gathered}
$$

such that

$$
|\widetilde{u}(t)-u(t)| \leq \lambda_{f} \varepsilon, t \in[a, b] .
$$

Definition 4 The equation (5) is generalized Ulam-Hyers stable if there exists $\varphi_{f} \in$ $C\left(\mathbb{R}^{+}, \mathbb{R}^{+}\right)$with $\varphi_{f}(0)=0$ such that for each $\varepsilon>0$ and for each solution $\widetilde{u} \in C^{n-1}[a, b]$ of the inequality (24) there exists a solution $u \in C^{n-1}[a, b]$ of problem (5)-(6) with

$$
|\widetilde{u}(t)-u(t)| \leq \varphi_{f}(\varepsilon), \quad t \in[a, b] .
$$

Lemma 5 [27] (generalized Gronwall's inequality) Let $u, v$, be two integrable functions and $h$ is continuous on $[a, b]$. Let $\psi \in C[a, b]$ be an increasing function such that $\psi^{\prime}(t) \neq 0, \forall t \in[a, b]$. Assume that $u$ and $v$ are nonnegative, $h$ is nonnegative and nondecreasing. If

$$
u(t) \leq v(t)+h(t) \int_{a}^{t} \psi^{\prime}(s)(\psi(t)-\psi(s))^{\alpha-1} u(s) d s,
$$

then, for all $t \in[a, b]$, we have

$$
u(t) \leq v(t)+\int_{a}^{t} \sum_{k=1}^{\infty} \frac{[h(t) \Gamma(\alpha)]^{k}}{\Gamma(\alpha k)} \psi^{\prime}(s)(\psi(t)-\psi(s))^{\alpha k-1} v(s) d s .
$$

Further, if $v$ is a nondecreasing function on $[a, b]$ then

$$
u(t) \leq v(t) E_{\alpha}\left[h(t) \Gamma(\alpha)(\psi(t)-\psi(a))^{\alpha}\right],
$$

where $E_{\alpha}(z)=\sum_{k=0}^{\infty} \frac{z^{k}}{\Gamma(\alpha k+1)},(z \in \mathbb{C})$ is called the Mittag-Leffler function.

Remark 1 A function $\widetilde{u} \in C^{n-1}[a, b]$ is a solution of the inequality (24) if and only if there exists a function $h \in C^{n-1}[a, b]$ (where $h$ depends on solution $\widetilde{u}$ ) such that

(i) $|h(t)| \leq \varepsilon$ for all $t \in[a, b]$, (ii) ${ }^{c} \mathscr{D}_{a^{+}}^{\alpha, \psi} \widetilde{u}(t)=f\left(t, \widetilde{u}(t), F_{\widetilde{u}}(t)\right)+h(t), \quad t \in[a, b] . \square$

Theorem 3 Under the assumptions of Theorem 1, the equation (5) is Ulam-Hyers and generalized Ulam-Hyers stable in $C^{n-1}[a, b]$. 
Proof In view of Theorem 1, the function $u \in C^{n-1}[a, b]$ is a unique solution of the problem (5)-(6) that is

$$
u(t)=\mathscr{A}_{u}+\frac{1}{\Gamma(\alpha)} \int_{a}^{t} \mathscr{R}_{\psi}^{\alpha-1}(t, s) f\left(s, u(s), F_{u}(s)\right) d s,
$$

where $\mathscr{R}_{\psi}^{\alpha-1}(t, s):=\psi^{\prime}(s)[\psi(b)-\psi(s)]^{\alpha-1}$ and

$$
\begin{aligned}
\mathscr{A}_{u}: & =\left[\frac{u_{b}}{(n-1) !}+\frac{f\left(a, u(a), F_{u}(a)\right)[\psi(b)-\psi(a)]^{\alpha-n+1}}{(n-2) ! \Gamma(\alpha-n+2)}\right][\psi(t)-\psi(a)]^{n-1} \\
& -\frac{[\psi(t)-\psi(a)]^{n-1}}{(n-1) ! \Gamma(\alpha-n+1)} \int_{a}^{b} \mathscr{R}_{\psi}^{\alpha-n}(b, s) f\left(s, u(s), F_{u}(s)\right) d s+\sum_{k=0}^{n-2} \frac{u_{a}^{k}}{k !}[\psi(t)-\psi(a)]^{k} .
\end{aligned}
$$

Let $\widetilde{u} \in C^{n-1}[a, b]$ is a solution of the inequality (24). By Remark 1, we have

$$
\left|\widetilde{u}(t)-\mathscr{A}_{\widetilde{u}}-\frac{1}{\Gamma(\alpha)} \int_{a}^{t} \mathscr{R}_{\psi}^{\alpha-1}(t, s) f\left(s, \widetilde{u}(s), F_{\widetilde{u}}(s)\right) d s\right| \leq \frac{[\psi(b)-\psi(a)]^{\alpha}}{\Gamma(\alpha+1)} \varepsilon,
$$

where

$$
\begin{aligned}
\mathscr{A}_{\widetilde{u}}: & =\left[\frac{u_{b}}{(n-1) !}+\frac{f\left(a, \widetilde{u}(a), F_{\widetilde{u}}(a)\right)[\psi(b)-\psi(a)]^{\alpha-n+1}}{(n-2) ! \Gamma(\alpha-n+2)}\right][\psi(t)-\psi(a)]^{n-1} \\
& -\frac{[\psi(t)-\psi(a)]^{n-1}}{(n-1) ! \Gamma(\alpha-n+1)} \int_{a}^{b} \mathscr{R}_{\psi}^{\alpha-n}(b, s) f\left(s, \widetilde{u}(s), F_{\widetilde{u}}(s)\right) d s+\sum_{k=0}^{n-2} \frac{u_{a}^{k}}{k !}[\psi(t)-\psi(a)]^{k} .
\end{aligned}
$$

Due to (26), $\mathscr{A} \widetilde{u}=\mathscr{A}_{u}$. Hence

$$
u(t)=\mathscr{A}_{\tilde{u}}+\frac{1}{\Gamma(\alpha)} \int_{a}^{t} \mathscr{R}_{\psi}^{\alpha-1}(t, s) f\left(s, u(s), F_{u}(s)\right) d s,
$$

From our assumption, we obtain

$$
\left|f\left(s, \widetilde{u}(s), F_{\widetilde{u}}(s)\right)-f\left(s, u(s), F_{u}(s)\right)\right| \leq \frac{\eta}{1-\eta}|\widetilde{u}(s)-u(s)| .
$$

Thus

$$
\begin{aligned}
& |\widetilde{u}(t)-u(t)| \\
\leq & \left|\widetilde{u}(t)-\mathscr{A}_{\widetilde{u}}-\frac{1}{\Gamma(\alpha)} \int_{a}^{t} \mathscr{R}_{\psi}^{\alpha-1}(t, s) f\left(s, \widetilde{u}(s), F_{\widetilde{u}}(s)\right) d s\right| \\
& +\frac{1}{\Gamma(\alpha)} \int_{a}^{t} \mathscr{R}_{\psi}^{\alpha-1}(t, s)\left|f\left(s, \widetilde{u}(s), F_{\widetilde{u}}(s)\right)-f\left(s, u(s), F_{u}(s)\right)\right| d s \\
\leq & \frac{[\psi(b)-\psi(a)]^{\alpha}}{\Gamma(\alpha+1)} \varepsilon+\frac{\eta}{1-\eta} \frac{1}{\Gamma(\alpha)} \int_{a}^{t} \mathscr{R}_{\psi}^{\alpha-1}(t, s)|\widetilde{u}(s)-u(s)| d s .
\end{aligned}
$$


It follows from Lemma 5 that

$$
\begin{aligned}
& |\widetilde{u}(t)-u(t)| \leq \frac{[\psi(b)-\psi(a)]^{\alpha}}{\Gamma(\alpha+1)} \varepsilon E_{\alpha}\left(\frac{\eta}{1-\eta}(\psi(t)-\psi(a))^{\alpha}\right) \\
& \leq \frac{[\psi(b)-\psi(a)]^{\alpha}}{\Gamma(\alpha+1)} \varepsilon E_{\alpha}\left(\frac{\eta}{1-\eta}(\psi(b)-\psi(a))^{\alpha}\right) \\
& \text { for } \lambda_{f}=\frac{[\psi(b)-\psi(a)]^{\alpha}}{\Gamma(\alpha+1)} E_{\alpha}\left(\frac{\eta}{1-\eta}(\psi(b)-\psi(a))^{\alpha}\right) \text { with } t \in[a, b] \text {, we get } \\
& |\widetilde{u}(t)-u(t)| \leq \lambda_{f} \varepsilon,
\end{aligned}
$$

This means that the problem (5)-(6) is Ulam-Hyers stable.

Theorem 4 Let the hypotheses of Theorem 3 hold. If there exists $\varphi_{f} \in C\left(\mathbb{R}^{+}, \mathbb{R}^{+}\right)$ with $\varphi_{f}(0)=0$. Then problem (5)-(6) has generalized Ulam-Hyers stability.

PROOF In a manner similar to Theorem 3, with choosing $\varphi_{f}(\varepsilon)=\lambda_{f} \varepsilon$ and $\varphi_{f}(0)=0$, we get

$$
|\widetilde{u}(t)-u(t)| \leq \varphi_{f}(\varepsilon)
$$

\section{An example}

Consider the implicit fractional differential equation

$$
\begin{gathered}
{ }^{c} \mathscr{D}_{0^{+}}^{\alpha ; \psi} u(t)=\frac{1}{2}\left(u(t)+{ }^{c} \mathscr{D}_{0^{+}}^{\alpha ; \psi} u(t)\right), t \in[0,1] \\
u_{\psi}^{[k]}(0)=0, k=0,1, u_{\psi}^{(2)}(1)=1,
\end{gathered}
$$

Here, $f\left(t, u(t),{ }^{c} \mathscr{D}_{0^{+}}^{\alpha ; \psi} u(t)\right)=\frac{1}{2}\left(u(t)+{ }^{c} \mathscr{D}_{0^{+}}^{\alpha ; \psi} u(t)\right),\left(t, u,{ }^{c} \mathscr{D}_{0^{+}}^{\alpha ; \psi} u\right) \in[0,1] \times \mathbb{R} \times \mathbb{R}$. Let $u_{i}, v_{i} \in \mathbb{R}(i=1,2)$ and $t \in[0,1]$. Then

$$
\left|f\left(t, u_{1}, v_{1}\right)-f\left(t, u_{2}, v_{2}\right)\right| \leq \frac{1}{2}\left[\left|u_{1}-u_{2}\right|+\left|v_{1}-v_{2}\right|\right] .
$$

So the condition (10) holds with $\eta=\frac{1}{2}$. We shall check that condition in (11) holds too. for example, $\alpha=\frac{5}{2}, n=\left[\frac{5}{2}\right]+1=3$, and $\psi(t)=\sqrt{t+1}$ for all $t \in[0,1]$, then upon computation we get

$$
\left[\frac{1}{\Gamma(\alpha+1)}+\frac{[\psi(b)-\psi(a)]+n-1}{(n-1) ! \Gamma(\alpha-n+2)}\right] \frac{\eta}{1-\eta}[\psi(b)-\psi(a)]^{\alpha} \approx 0.184<1 .
$$


Thus, by Theorem 1, the problem (29)-(30) has a unique solution on $[0,1]$.

Further, by the application of Theorem 2, it is easy to see that condition (19) holds with $k_{0}=k_{1}=\frac{1}{2}$, i.e. $|f(t, u, v)| \leq \frac{1}{2}|u|+\frac{1}{2}|v|$ for $(t, u, v) \in[0,1] \times \mathbb{R} \times \mathbb{R}$. Also the inequality (20) is satisfied, i.e.

$$
\frac{k_{0}}{1-k_{1}}[\psi(b)-\psi(a)]^{\alpha}\left[\frac{1}{\Gamma(\alpha+1)}+\frac{n}{(n-1) ! \Gamma(\alpha-n+2)}\right] \approx 0.220<1 .
$$

Therefore, Theorem 2 shows that the problem (29)-(30) has a solution on $[0,1]$. On the other hand, the problem (29)-(30) is stable in the sense of Ulam-Hyers with

$$
|\widetilde{u}(t)-u(t)| \leq \frac{(\sqrt{2}-1)^{\frac{5}{2}}}{\Gamma\left(\frac{7}{2}\right)} E_{\frac{5}{2}}\left((\sqrt{2}-1)^{\frac{5}{2}}\right) \varepsilon, \quad t \in[0,1] .
$$

\section{Conclusions}

We have provided sufficient conditions ensuring the existence, uniqueness and Ulam-Hyers stability of the solutions to a class of a boundary value problem for implicit fractional differential equations involving a general form of the Caputo fractional derivative with respect to another function $\psi$. The proofs rely on Banach's fixed point theorem, Schauder's fixed point theorem, generalized Gronwall's inequality and some important results within the mathematical analysis. As an example of future work, one can generalize existence results in an impulsive fractional problem, a neutral time delay problem, and a time-delay problem with finite and infinite delay.

Acknowledgments. The authors would like to thank the referees and editors for their careful reading of the work and insightful comments, which helped improve the quality of the paper.

\section{References}

[1] Hilfer, R. (2000). Applications of Fractional Calculus in Physics. Singapore: World Scientific.

[2] Kilbas, A.A., Srivastava, H.M., \& Trujillo, J.J. (2006). Theory and Applications of Fractional Differential Equations. Amsterdam: North-Holland Math. Stud, Elsevier.

[3] Magin, R.L. (2006). Fractional Calculus in Bioengineering. Begell House Inc. Publisher.

[4] Samko, S.G., Kilbas A.A., \& Marichev O.I. (1993). Fractional Integrals and Derivatives, Theory and Applications. Yverdon: Gordon and Breach.

[5] Abdo, M.S., \& Panchal, S.K. (2019). Fractional integro-differential equations involving $\psi$-Hilfer fractional derivative. Adv. Appl. Math. Mech., 11(1), 1-22.

[6] Abdo, M.S., \& Panchal, S.K., (2018). Weighted fractional neutral functional differential equations. J. Siber. Fed. Univ. Math. Phys., 11(5), 1-15. 
[7] Agarwal, R. Hristova, S., \& O'Regan, D. (2016). A survey of Lyapunov functions, stability and impulsive Caputo fractional differential equations. Fract. Calc. Appl. Anal., 19(2), 290-318.

[8] Al-Saqabi, B., \& Kiryakova, V.S. (1998). Explicit solutions of fractional integral and differential equations involving Erdelyi-Kober operators. Appl. Math. Comput., 95(1), 1-13.

[9] Kucche, K.D., Nieto, J.J., \& Venktesh, V. (2016). Theory of nonlinear implicit fractional differential equations. Diff. Equ. Dynam. Sys., 1-17.

[10] Li, M., \& Wang, J. (2015). Existence of local and global solutions for Hadamard fractional differential equations. Electron. J. Differ. Equ., 2015(166), 1-8.

[11] Sun, Y., Zeng, Z., \& Song, J. (2017). Existence and uniqueness for the boundary value problems of nonlinear fractional differential equation. Appl. Math., 8(3), 312.

[12] Ulam, S.M. (1968). A Collection of Mathematical Problems. New York: Interscience.

[13] Hyers, D.H. (1941). On the stability of the linear functional equation. Proc. Natl. Acad. Sci., 27, 222-224.

[14] Andras, S. \& Kolumban, J.J. (2013). On the Ulam-Hyers stability of first order differential systems with nonlocal initial conditions. Nonlinear Analysis: Theory, Methods \& Applications, $82,1-11$.

[15] Benchohraa, M., \& Bouriaha, S. (2015). Existence and stability results for nonlinear boundary value problem for implicit differential equations of fractional order. Moroccan. J. Pure. Appl. Anal., 1(1), 22-37.

[16] Ibrahim, R.W. (2012). Generalized Ulam-Hyers stability for fractional differential equations. International Journal of Mathematics, 23, 1-9.

[17] Jung, S.M. (2004). Hyers-Ulam stability of linear differential equations of first order. Applied Mathematics Letters, 17, 1135-1140.

[18] Almeida, R. (2017). A Caputo fractional derivative of a function with respect to another function. Commun. Nonlinear Sci. Numer. Simul., 44, 460-481.

[19] Ardjouni, A., \& Djoudi, A. (2019). Existence and uniqueness of solutions for nonlinear implicit Caputo-Hadamard fractional differential equations with nonlocal conditions. Advances in the Theory of Nonlinear Analysis and its Applications, 3(1), 46-52.

[20] Dong, J., Feng, Y., \& Jiang, J. (2017). A note on implicit fractional differential equations. Mathematica Aeterna, 7(3), 261-267.

[21] Haoues, M., Ardjouni, A., \& Djoudi, A. (2018). Existence, interval of existence and uniqueness of solutions for nonlinear implicit Caputo fractional differential equations. TJMM, 10(1), 09-13.

[22] Nieto, J., Ouahab, A., \& Venktesh, V. (2015). Implicit fractional differential equations via the Liouville-Caputo derivative. Mathematics, 3(2), 398-411.

[23] Almeida, R. (2019). Fractional differential equations with mixed boundary conditions. Bulletin of the Malaysian Mathematical Sciences Society, 42(4), 1687-1697.

[24] Almeida, R., Malinowska, A.B., \& Monteiro, M.T. (2018). Fractional differential equations with a Caputo derivative with respect to a kernel function and their applications. Math. Meth. Appl. Sci., 41(1), 336-352.

[25] Vivek D., Elsayed E.M., \& Kanagarajan K. (2018). Theory and analysis of $\psi$-fractional differential equations with boundary conditions. Communications in Applied Analysis, 22(3), 401-414.

[26] Abdo, M.S., Panchal, S.K., \& Abdulkafi M. Saeed (2019). Fractional boundary value problem with $\psi$-Caputo fractional derivative. Proc. Indian Acad. Sci. (Math. Sci.), 129, 65.

[27] Sousa, J.V.C., \& Oliveira, E.C. (2019). A Gronwall inequality and the Cauchy-type problem by means of $\psi$-Hilfer operator. Diff. Equ. Appl., 11(1), 87-106. 